\title{
ASYMPTOTIC ANALYSIS OF A MULTIPHASE DRYING MODEL MOTIVATED BY COFFEE BEAN ROASTING *
}

\author{
NABIL T. FADAI ${ }^{\dagger}$, COLIN P. PLEASE ${ }^{\dagger}$, AND ROBERT A. VAN GORDER ${ }^{\dagger}$
}

\begin{abstract}
Recent modelling of coffee bean roasting suggests that in the early stages of roasting, within each coffee bean, there are two emergent regions: a dried outer region and a saturated interior region. The two regions are separated by a transition layer (or, drying front). In this paper, we consider the asymptotic analysis of a recent multiphase model in order to gain a better understanding of its salient features. The model consists of a PDE system governing the thermal, moisture, and gas pressure profiles throughout the interior of the bean. By obtaining asymptotic expansions for these quantities in relevant limits of the physical parameters, we are able to determine the qualitative behaviour of the outer and interior regions, as well as the dynamics of the drying front. Although a number of simplifications and scalings are used, we take care not to discard aspects of the model which are fundamental to the roasting process. Indeed, we find that for all of the asymptotic limits considered, our approximate solutions faithfully reproduce the qualitative features evident from numerical simulations of the full model. From these asymptotic results, we have a better qualitative understanding of the drying front (which is hard to resolve precisely in numerical simulations), and hence, of the various mechanisms at play including heating, evaporation, and pressure changes. This qualitative understanding of solutions to the multiphase model is essential when creating more involved models that incorporate chemical reactions and solid mechanics effects.
\end{abstract}

Key words. multiphase model, coffee bean roasting, Stefan problem, asymptotic analysis, drying front

AMS subject classifications. 80A22, 80M $35,74 \mathrm{~N} 20,82 \mathrm{C} 26$

1. Introduction. As one of the most valuable commodities in the world [1], the coffee industry relies on fundamental research to improve the techniques and processes relating to its products, especially with the roasting process of coffee beans. Most of the literature concerning the roasting of coffee beans present experimental data (see e.g. [2,3,4]) and use regression analysis and simple empirical models to interpret the results. Recently, the literature has included a more in-depth discussion concerning the mathematical modelling of the roasting of coffee beans (see e.g. [5,6]). While other aspects of coffee processing have been examined from a mathematical perspective (e.g. [7]), mathematical models describing the roasting of coffee beans have, with the exception of a few studies, been largely unexplored.

In [5], a system of partial differential equations (PDEs) that model the transport of moisture and heat throughout a coffee bean were derived and studied. This model uses the concept of "mass diffusivity" to describe the transport of moisture in the coffee bean that was originally derived in [8], which applies to lower-temperature evaporation. The ideas in [5] served as excellent motivation for the authors in [6] to derive a mathematical model from first principles using conservation equations. Multiphase modelling has been previously been applied in a variety of food heating problems $[9,10,11,12,13,14]$ (of particular relevance was the bread baking model of Zhang et al. [15]) and is a natural framework to model the coffee bean roasting process. In [6], the concepts of multiphase flow and water evaporation were included, and the resulting model (referred to as Model 2 in [6]) also incorporated the production of carbon dioxide gas, latent heat due to evaporation, and changes in porosity. Some simplifications were made to this full model (in particular, neglecting carbon dioxide production) in order to allow some preliminary understanding of the model behaviour. By examining numerical solutions of this model, a "drying front" that propagates through to the center of the bean was identified.

Moisture transport in roasting coffee beans is a crucial aspect of the industrial process. Many chemical reactions that occur produce aromas and flavour compounds that determine the quality of the final product. Some of these chemical reactions are believed to be affected by the presence (or lack) of water [3]. Therefore, the spatial and temporal distribution of the moisture content is strongly linked to the quality of the final product.

Mathematical models describing drying have been explored previously (see e.g. [16, 17, 18]), which relate the drying of wood, bricks, and other materials. However, due to the impermeable cellulose structure within a coffee bean, the water vapour created in the biological cells cannot be easily released into the roasting environment. In consequence, the ratio in timescales between evaporation dynamics and vapour transport is

*This publication is based on work supported by the EPSRC center for Doctoral Training in Industrially Focused Mathematical Modelling (EP/L015803/1)

${ }^{\dagger}$ Mathematical Institute, University of Oxford, Andrew Wiles Building, Radcliffe Observatory Quarter, Woodstock Road, Oxford, OX2 6GG, United Kingdom (Robert.VanGorder@maths.ox.ac.uk) 
very large, which motivates us to explore the leading-order dynamics of coffee bean roasting using asymptotic analysis. Hence, the model presented here should be appropriate to any drying problem where these physical phenomena are relevant.

In [6], numerical results suggest that there are three main regions within a coffee bean as it is roasted. The first main region (which we refer to as Region i) is where the vapour pressure of water aligns with the steam table pressure. The second main region (which we refer to as Region ii) is when the moisture content of the bean is negligible. Between these two regions, we expect a thin transition layer, or "drying front", in which moisture is rapidly evaporated. Issues surrounding numerical resolution make it computationally expensive to resolve the dynamics near the drying front. In light of these observations, we are motivated to extend the numerical results shown in [6] via asymptotic methods, in order to understand the qualitative features of the multiphase model: in particular, the interplay between the narrow transition layer and the two larger regions.

In this paper, we begin our discussion of the asymptotics of the full multiphase model in Section 2. Motivated by the numerical results in [6], we determine an approximate form of the drying front. We then obtain the leading-order asymptotics in Regions i and ii, as well as within the drying front. Despite several simplifications, we are able to obtain reasonable agreement between the asymptotic approximations and the numerical solutions in [6], and are confident that the asymptotics capture the qualitative dynamics of the problem. In order to obtain additional explicit results, and motivated by the observation that the entire coffee bean is almost always very close to the externally imposed roasting temperature, we fix the temperature at this roasting temperature in Section 3. Under this assumption, the vapour pressure and moisture content are also constant in Region i, while the leading order dynamics within Region ii reduce to a Stefan problem [19]. By considering the large Stefan number limit, we determine a leading-order expression for the drying front for various geometries. As we will only focus on symmetric geometries with one spatial variable (e.g. planar and spherical geometries), we can obtain explicit expressions for the drying front. We focus on the planar and spherical geometries, as it is reasonable to represent a coffee bean either as a slab of porous material "curled up" into the shape of a bean or as a sphere. Finally, in Sec. 4, we provide a summary and discussion of the results.

2. Asymptotics of the Multiphase Model with Variable Temperature. The full multiphase model that we will analyse consists of three PDEs that describe conservation of mass in water and vapour, as well as conservation of energy. These equations describe the behaviour of three variables: the water saturation $S$ (i.e. the volume fraction of water divided by the total volume of water and gas), the partial pressure of water vapour $P$, and the temperature $T$. The only transport mechanism considered for water is via evaporation, whereas in the gas phase, water vapour is transported either via evaporation or via Darcy flow. Finally, we assume that heat is transported via conduction in all three phases within the bean, but via convection at the surface of the bean. Mathematically speaking, this multiphase model can be stated for nondimensional variables in symmetric geometries (i.e. using a single spatial variable $r$ ) as

$$
\begin{aligned}
\frac{\partial S}{\partial t} & =-\frac{1}{\epsilon^{2}} I_{v}, \\
\frac{\partial}{\partial t}\left[\frac{(1+\mathscr{T}) P(1-\sigma S)}{1+\mathscr{T} T}\right] & =-\frac{1}{\delta} \frac{\partial S}{\partial t}+\nabla \cdot\left[\frac{(1+\mathscr{T}) P \nabla P}{1+\mathscr{T} T}\right], \\
\frac{\partial T}{\partial t}+\mathcal{A}_{1} \frac{\partial}{\partial t}[S(1+\mathscr{T} T)] & =\mathcal{A}_{2} \frac{\partial S}{\partial t}+\mathcal{A}_{3} \nabla \cdot\left[\left(1+\mathcal{A}_{4} S\right) \nabla T\right],
\end{aligned}
$$

where the $\nabla$ operator should be interpreted appropriately for the different geometries as derivatives of $r$. Additionally, there are symmetry conditions at the centre of the bean

$$
\left.\nabla T \cdot \mathbf{n}\right|_{r=0}=0,\left.\quad \nabla P \cdot \mathbf{n}\right|_{r=0}=0,
$$

the boundary conditions at the surface of the bean

$$
\left.\nabla T \cdot \mathbf{n}\right|_{r=1}=\nu\left(\frac{1-\sigma S}{1-\sigma}\right)\left(\frac{1+\mathcal{A}_{4}}{1+\mathcal{A}_{4} S}\right)(1-T),
$$

$$
\left.P\right|_{r=1}= \begin{cases}P_{S T}(T), & T<T_{a}, \\ P_{a}, & T \geq T_{a},\end{cases}
$$


and the initial conditions

$$
S(r, 0)=1, \quad T(r, 0)=0, \quad P(r, 0)=P_{S T}(0) .
$$

Here, the evaporation rate $I_{v}$ and steam table pressure $P_{S T}(T)$ are given by

$$
I_{v}=S(1-\sigma S)\left(P_{S T}-P\right) \sqrt{\frac{1+\mathscr{T}}{1+\mathscr{T} T}} \quad \text { and } \quad P_{S T}(T)=\exp \left(\frac{\beta(T-1)}{1+\mathscr{T} T}\right) .
$$

A complete derivation of this model from the "simplified" multiphase model presented in [6] can be found in Appendix A. A key feature of this model is $\epsilon$, which can be interpreted as a ratio in timescales between Darcy-driven vapour transport and evaporation. One can interpret $\delta$ as a density ratio of water vapour to water, and $\sigma$ represents the initial water-to-void volume ratio. The boundary condition (6) is slightly modified from that in [6] and is described in Appendix A. Here, $P_{a}$ is the ambient vapour pressure in the roasting chamber.

We will also make the assumption that the step in the boundary condition (6) for $P$ only occurs at one critical time, namely, $t^{*}$. We define $t^{*}$ as the first time when the evaporation temperature $T_{a}$ is achieved at the surface of the bean, i.e. as the solution to the equation

$$
T\left(1, t^{*}\right)=T_{a}:=P_{S T}^{-1}\left(P_{a}\right) .
$$

This critical time will be used not only to signal which part of the step in (6) is relevant, but also to signal where the asymptotic behaviour changes.

We can divide the solution to the model into three regions in order to understand the approximate dynamics that occur in the coffee bean. Using parameter values shown in [6], a typical value of $\epsilon \approx 1.54 \times 10^{-4}$ suggests that we should consider the limit of $\epsilon \rightarrow 0^{+}$. We note that $\delta=O(1)$ is the distinguished limit of this system and concentrate on considering this case, despite the analysis being also valid for small $\delta$. We will consider $\delta$ being small when the equations in Regions i and ii require further simplifications to extract closed form solutions. In Section 2.2, we will determine how small $\delta$ is allowed to be before a different analysis is required. In the $\epsilon \rightarrow 0^{+}$limit, we can see from (1) that if time and space remain unscaled, $I_{v}=0$ will be the leading-order equation, and from (8), this can occur in one of three ways. Firstly, $I_{v}=0$ if the vapour pressure is in equilibrium with its steam table pressure, i.e. $P=P_{S T}$. As the initial data is consistent with this equilibrium, we will observe this first (which will be referred to as Region i). Secondly, $I_{v}=0$ can be achieved by setting $S=0$. This corresponds to where there is no more water to evaporate and will be denoted as Region ii. A final case where $I_{v}=0$ is when $S=\sigma^{-1}$; however, this corresponds to when the coffee bean is completely saturated with water, which we will discard as an extraneous case.

We will also consider a narrow "drying front" that connects Regions i and ii. This drying front, which is centred about $r=R(t)$, propagates from the surface of the bean towards the center of the bean and is where the moisture content $S$ quickly goes from 1 to 0 . In this drying front around $R(t)$, we find that the temperature is spatially uniform, but will vary as time progresses. The temperature profile within the drying front is denoted as $T^{*}(t)$. A schematic diagram of these three regions is shown in Figure 1, including the time $t^{*}$ at which evaporation first occurs at the surface of the bean.

2.1. Asymptotics of Region i. In Region i, we have, from (1), $I_{v}=0$ to leading order in the limit of $\epsilon \rightarrow 0^{+}$, implying that that $P=P_{S T}(T)$. Consider the asymptotic series valid as $\epsilon \rightarrow 0^{+}$,

(10) $S=S_{0}(r, t)+\epsilon S_{1}(r, t)+O\left(\epsilon^{2}\right), \quad T=T_{0}(r, t)+\epsilon T_{1}(r, t)+O\left(\epsilon^{2}\right), \quad P=P_{S T}(r, t)+\epsilon P_{1}(r, t)+O\left(\epsilon^{2}\right)$.

Substituting (10) into (2) and (3) gives, to lowest order,

$$
\begin{aligned}
\frac{\partial S_{0}}{\partial t}\left(\frac{1}{\delta}-\sigma P_{S T}\left(T_{0}\right) \Lambda\left(T_{0}\right)\right)+\left(1-\sigma S_{0}\right) \frac{\partial}{\partial t}\left[P_{S T}\left(T_{0}\right) \Lambda\left(T_{0}\right)\right] & =\nabla \cdot\left[P_{S T}\left(T_{0}\right) \Lambda\left(T_{0}\right) \nabla P_{S T}\left(T_{0}\right)\right], \\
\frac{\partial S_{0}}{\partial t}\left(\mathcal{A}_{1}\left(1+\mathscr{T} T_{0}\right)-\mathcal{A}_{2}\right)+\frac{\partial T_{0}}{\partial t}\left[1+\mathcal{A}_{1} \mathscr{T} S_{0}\right] & =\mathcal{A}_{3} \nabla \cdot\left[\left(1+\mathcal{A}_{4} S_{0}\right) \nabla T_{0}\right],
\end{aligned}
$$

where $\Lambda\left(T_{0}\right)=\frac{1}{1+\mathscr{T} T_{0}}$. As we cannot solve this system analytically, we now suppose that $\delta \ll 1$ and write an asymptotic series in powers of $\delta$ for $S_{0}$ and $T_{0}$ valid in the limit $\delta \rightarrow 0^{+}$as

$$
S_{0}=\tilde{S}_{0}(r, t)+\delta \tilde{S}_{1}(r, t)+O\left(\delta^{2}\right), \quad T_{0}=\tilde{T}_{0}(r, t)+\delta \tilde{T}_{1}(r, t)+O\left(\delta^{2}\right) .
$$




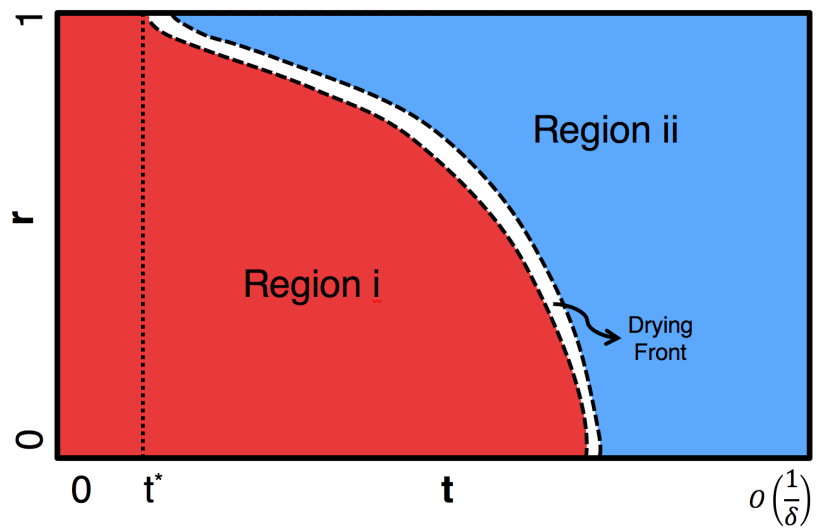

FIG. 1. A summary of where the different regions are as the bean dries. Region is when the vapour pressure is in equilibrium, Region ii is the dry region, and the dashed lines indicate the narrow transition layer between the regions, which begins at time $t^{*}$, defined in (9).

Substituting (13) into (11) gives us, to leading order, that $\frac{\partial \tilde{S}_{0}}{\partial t}=0$. Therefore, the moisture content of the bean stays at its initial value, i.e. $\tilde{S}_{0}=1$. To lowest order, (12) then gives us

$$
\frac{\partial \tilde{T}_{0}}{\partial t}=\mathcal{K} \nabla^{2} \tilde{T}_{0}, \quad \text { where } \quad \mathcal{K}=\frac{\mathcal{A}_{3}\left(1+\mathcal{A}_{4}\right)}{1+\mathcal{A}_{1} \mathscr{T}} .
$$

Equation (14) must be solves subject to the boundary condition (6), which can be stated as

$$
\begin{aligned}
\left.\nabla \cdot \tilde{T}_{0}\right|_{r=1} & =\nu\left(1-\left.\tilde{T}_{0}\right|_{r=1}\right), \quad t<t^{*}, \\
\left.\tilde{T}_{0}\right|_{r=R(t)} & =T^{*}(t), \quad t \geq t^{*} .
\end{aligned}
$$

Additionally, we will impose the symmetry condition $\nabla \tilde{T}_{0} \cdot \mathbf{n}=0$ at $r=0$, as well as the initial data $\tilde{T}_{0}(r, 0)=0$. We are able to solve the PDE for $t<t^{*}$; in particular, we can determine a leading-order approximation for $t^{*}$. By solving (14) in spherical co-ordinates, we obtain that

$$
\tilde{T}_{0}(r, t)=1-\sum_{n=1}^{\infty} \frac{c_{n}}{r} \sin \left(\mu_{n} r\right) \exp \left(-\mu_{n}^{2} \mathcal{K} t\right) .
$$

where the eigenvalues $\mu_{n}$ satisfy the transcendental equation $\mu_{n} \cot \left(\mu_{n}\right)=1-\nu$ and the constants $c_{n}$ have the form

$$
c_{n}= \begin{cases}\frac{2 \nu \cos \mu_{n}}{\mu_{n}\left(\sin ^{2} \mu_{n}-\nu\right)}, & \nu \neq 1, \\ \frac{8(-1)^{n}}{\pi^{2}(1+2 n)^{2}}, & \nu=1 .\end{cases}
$$

While the case $\nu=1$ is reasonably pathological, it is included in this analysis for completeness. Typical parameter values seen in [6] give $\nu \approx 0.585$, so we will only consider the case where $\nu \neq 1$ from here on.

To determine $t^{*}$ in spherical co-ordinates, denoted as $t_{\mathrm{Sph}}^{*}$, we impose, from (9), that $\tilde{T}_{0}\left(1, t_{\mathrm{Sph}}^{*}\right)=T_{a}$. When $\nu \neq 1$, this is equivalent to writing

$$
\sum_{n=1}^{\infty}\left(\frac{\cos ^{2} \mu_{n}}{\sin ^{2} \mu_{n}-\nu}\right) \exp \left(-\mu_{n}^{2} \mathcal{K} t_{\mathrm{Sph}}^{*}\right)=\frac{\left(1-T_{a}\right)(1-\nu)}{2 \nu} .
$$

Using the parameter values in $[6]\left(\mathcal{K} \approx 2.25, \nu \approx 0.585, T_{a} \approx 0.519\right),(19)$ has the solution $t_{\mathrm{Sph}}^{*} \approx 0.173$, or about 45.9 seconds in dimensional units.

Similarly, we can determine $t^{*}$ in Cartesian co-ordinates, denoted as $t_{\text {Cart }}^{*}$, by noting that the solution of (14) in Cartesian co-ordinates, with a Neumann boundary condition at $r=0$, is

$$
\tilde{T}_{0}(r, t)=1-\sum_{n=1}^{\infty} d_{n} \cos \left(\lambda_{n} r\right) \exp \left(-\lambda_{n}^{2} \mathcal{K} t\right)
$$


where

$$
\lambda_{n} \tan \lambda_{n}=\nu \quad \text { and } \quad d_{n}=\frac{2 \nu \sin \lambda_{n}}{\lambda_{n}\left(\nu+\sin ^{2} \lambda_{n}\right)}
$$

This allows us to determine $t_{\text {Cart }}^{*}$ via the transcendental equation

$$
\sum_{n=1}^{\infty} \frac{\sin ^{2} \lambda_{n}}{\nu+\sin ^{2} \lambda_{n}} \exp \left(-\lambda_{n}^{2} \mathcal{K} t_{\text {Cart }}^{*}\right)=\frac{1-T_{a}}{2}
$$

which, using parameter values stated above, gives $t_{\text {Cart }}^{*} \approx 0.494$, or about 131 seconds in dimensional units. Hence, we have determined when the step occurs in the boundary condition (6). When the boundary condition changes, we find that the transition layer forms at the surface. We shall not consider the very small time while the transition layer is close to the surface, as it does not give any useful insight, but proceed to when it is in the interior of the bean.

2.2. Asymptotics of the Transition Layer. In order to understand how $S$ varies from 1 to 0 , we must examine the transition layer in the limit $\epsilon \rightarrow 0^{+}$. We first discuss the distinguished limit $\delta=O(1)$. We will find that both $P$ and $T$ are spatially uniform at lowest order with small variations of equal size (say $O\left(\epsilon^{a}\right), a>0$ ) and hence variations of the evaporation rate are also $O\left(\epsilon^{a}\right)$. The transition layer is narrow (say $\left.O\left(\epsilon^{b}\right), b>0\right)$ around a moving front at $r=R(t)$ and we find, noting that $S=O(1)$, that a balance between the resulting advective term in (1) and the evaporation rate requires $-b=-2+a$. Similarly, having a balance of vapour production due to evaporation with transport by Darcy flow in (2) requires that $-b=-2 b+a$. Hence, in the distinguished limit $\delta=O(1)$, we take $a=b=1$. We can also consider small $\delta$; when $\delta=O\left(\epsilon^{\alpha}\right)$ and $\alpha>0$, a dominant balance requires that $-b=a-2$ and $-\alpha-b=-2 b+a$. Therefore, the transition layer has width $O\left(\epsilon^{1+\alpha / 2}\right)$ and the variations in $P$ and $T$ are $O\left(\epsilon^{1-\alpha / 2}\right)$. Since we require these variations to be small, we must have $\alpha<2$. We conclude that the analysis in the distinguished limit $\delta=O(1)$ is valid until $\delta=O\left(\epsilon^{2}\right)$.

For the case $\delta=O(1)$, the transition layer is around "drying front" $R(t)$ and we introduce the scaling $r=R(t)+\epsilon \hat{r}$. We also take $P, T$, and $S$ as asymptotic series as $\epsilon \rightarrow 0^{+}$, with

$$
S=S_{0}(\hat{r}, t)+\epsilon S_{1}(\hat{r}, t)+O\left(\epsilon^{2}\right), \quad P=P_{0}(\hat{r}, t)+\epsilon P_{1}(\hat{r}, t)+O\left(\epsilon^{2}\right), \quad T=T_{0}(\hat{r}, t)+\epsilon T_{1}(\hat{r}, t)+O\left(\epsilon^{2}\right) .
$$

We will first show that $T_{0}(\hat{r}, t) \equiv T^{*}(t)$ and $P_{0}(\hat{r}, t) \equiv P^{*}(t):=P_{S T}\left(T^{*}(t)\right)$. To do this, we note that, in order to match our transition layer into Region $\mathrm{i}$, we must have that

$$
\left.P_{0}\right|_{\hat{r} \rightarrow-\infty} \rightarrow P^{*}(t) \text { and }\left.\quad T_{0}\right|_{\hat{r} \rightarrow-\infty} \rightarrow T^{*}(t) .
$$

By substituting (23) into (2) and (3), we obtain, at $O\left(\epsilon^{-2}\right)$,

$$
\frac{\partial}{\partial \hat{r}}\left[\frac{P_{0} \frac{\partial P_{0}}{\partial \hat{r}}}{1+\mathscr{T} T_{0}}\right]=0, \quad \frac{\partial}{\partial \hat{r}}\left[\frac{\partial T_{0}}{\partial \hat{r}}\left(1+\mathcal{A}_{4} S_{0}\right)\right]=0 .
$$

We note that these equations hold in any geometry at leading order, provided that we are sufficiently far away from any geometry-induced singularities that could produce additional derivative terms at $O\left(\epsilon^{-2}\right)$, e.g. if $R(t)=O(\epsilon)$ in spherical co-ordinates. Integrating (25) and imposing (24) implies that

$$
T_{0}(\hat{r}, t) \equiv T^{*}(t) \quad \text { and } \quad P_{0}(\hat{r}, t) \equiv P^{*}(t) .
$$

To determine the leading-order behaviour for $S$, we note that using (23) in (8) and expanding yields

$$
\begin{array}{r}
P_{S T}=P^{*}\left(1+\epsilon \frac{\beta(1+\mathscr{T})}{\left(1+\mathscr{T} T^{*}\right)^{2}} T_{1}\right)+O\left(\epsilon^{2}\right), \\
I_{v}=-\epsilon\left(P_{1}-\frac{\beta(1+\mathscr{T})}{\left(1+\mathscr{T} T^{*}\right)^{2}} T_{1} P^{*}\right) S_{0}\left(1-\sigma S_{0}\right) \sqrt{\frac{1+\mathscr{T}}{1+\mathscr{T} T^{*}}}+O\left(\epsilon^{2}\right) .
\end{array}
$$


Using these along with (24), we obtain, at $O\left(\epsilon^{-1}\right)$, that (1)-(3) give

$$
\begin{aligned}
-R^{\prime}(t) \frac{\partial S_{0}}{\partial \hat{r}} & =\Psi\left(P_{1}, T_{1}\right) S_{0}\left(1-\sigma S_{0}\right), \\
\sigma P^{*} R^{\prime}(t) \frac{\partial S_{0}}{\partial \hat{r}} & =-\frac{1}{\delta} \Psi\left(P_{1}, T_{1}\right) S_{0}\left(1-\sigma S_{0}\right)\left(\frac{1+\mathscr{T} T^{*}}{1+\mathscr{T}}\right)+P^{*} \frac{\partial^{2} P_{1}}{\partial \hat{r}^{2}} \\
-\mathcal{A}_{1}\left(1+\mathscr{T} T^{*}\right) R^{\prime}(t) \frac{\partial S_{0}}{\partial \hat{r}} & =\mathcal{A}_{2} \Psi\left(P_{1}, T_{1}\right) S_{0}\left(1-\sigma S_{0}\right)+\mathcal{A}_{3} \frac{\partial}{\partial \hat{r}}\left[\left(1+\mathcal{A}_{4} S_{0}\right) \frac{\partial T_{1}}{\partial \hat{r}}\right],
\end{aligned}
$$

where

$$
\Psi\left(P_{1}, T_{1}\right):=\sqrt{\frac{1+\mathscr{T}}{1+\mathscr{T} T^{*}}}\left(P_{1}-\frac{\beta(1+\mathscr{T})}{\left(1+\mathscr{T} T^{*}\right)^{2}} T_{1} P^{*}\right)
$$

Finally, the matching conditions with Regions i and ii are

$$
\begin{aligned}
& S_{0} \rightarrow 1, \quad P_{1} \rightarrow 0, \text { and } T_{1} \rightarrow 0 \text { as } \hat{r} \rightarrow-\infty \text {, } \\
& S_{0} \rightarrow 0 \text { as } \hat{r} \rightarrow+\infty \text {, } \\
& \left.\frac{\partial P_{1}}{\partial \hat{r}}\right|_{\hat{r} \rightarrow+\infty}=\left.\frac{\partial P}{\partial r}\right|_{r \rightarrow R(t)}, \\
& \left.\frac{\partial T_{1}}{\partial \hat{r}}\right|_{\hat{r} \rightarrow+\infty}=\left.\frac{\partial T}{\partial r}\right|_{r \rightarrow R(t)} \text {. }
\end{aligned}
$$

In interpreting (32)-(35), we note that the limits where $r \rightarrow R(t)$ are matching conditions for Regions $\mathrm{i}$ and ii, whereas the limits where $\hat{r} \rightarrow \pm \infty$ refer to matching conditions for the transition layer.

By eliminating the terms with $\Psi\left(P_{1}, T_{1}\right)$ in (28) and (29), we obtain

$$
P^{*} \frac{\partial^{2} P_{1}}{\partial \hat{r}^{2}}=\left[\sigma P^{*}-\frac{1}{\delta}\left(\frac{1+\mathscr{T} T^{*}}{1+\mathscr{T}}\right)\right] R^{\prime}(t) \frac{\partial S_{0}}{\partial \hat{r}} .
$$

Integrating this and imposing the matching conditions (32) yields

$$
\frac{\partial P_{1}}{\partial \hat{r}}=\left[\frac{1}{\delta}\left(\frac{1+\mathscr{T} T^{*}}{(1+\mathscr{T}) P^{*}}\right)-\sigma\right] R^{\prime}(t)\left(1-S_{0}\right) .
$$

Similarly, eliminating terms with $\Psi\left(P_{1}, T_{1}\right)$ in (28) and (30) gives us

$$
R^{\prime}(t)\left[\mathcal{A}_{2}-\mathcal{A}_{1}\left(1+\mathscr{T} T^{*}\right)\right] \frac{\partial S_{0}}{\partial \hat{r}}=\mathcal{A}_{3} \frac{\partial}{\partial \hat{r}}\left[\left(1+\mathcal{A}_{4} S_{0}\right) \frac{\partial T_{1}}{\partial \hat{r}}\right] .
$$

Integrating and imposing the matching conditions (32) yields, after some rearranging,

$$
\frac{\partial T_{1}}{\partial \hat{r}}=-\frac{1}{\mathcal{A}_{3}} R^{\prime}(t)\left[\mathcal{A}_{2}-\mathcal{A}_{1}\left(1+\mathscr{T} T^{*}\right)\right]\left(\frac{1-S_{0}}{1+\mathcal{A}_{4} S_{0}}\right) .
$$

Finally, by rearranging (28) to isolate $S_{0}$, we obtain

$$
\frac{\frac{\partial S_{0}}{\partial \hat{r}}}{S_{0}\left(1-\sigma S_{0}\right)}=-\frac{\Psi\left(P_{1}, T_{1}\right)}{R^{\prime}(t)} .
$$

In order to write a single differential equation for $S_{0}$, we differentiate (40) with respect to $\hat{r}$, as well as substitute in (37) and (39), to give us

$$
\frac{\partial^{2} S_{0}}{\partial \hat{r}^{2}}-\left(\frac{\partial S_{0}}{\partial \hat{r}}\right)^{2} \frac{1-2 \sigma S_{0}}{S_{0}\left(1-\sigma S_{0}\right)}+S_{0}\left(1-\sigma S_{0}\right)\left(1-S_{0}\right) \Upsilon\left(S_{0}\right)=0,
$$


where we define

$$
\Upsilon\left(S_{0}\right):=\sqrt{\frac{1+\mathscr{T}}{1+\mathscr{T} T^{*}}}\left[\frac{1}{\delta}\left(\frac{1+\mathscr{T} T^{*}}{(1+\mathscr{T}) P^{*}}\right)-\sigma-\left(\frac{\beta(1+\mathscr{T}) P^{*}}{\mathcal{A}_{3}\left(1+\mathscr{T} T^{*}\right)^{2}}\right)\left(\frac{\mathcal{A}_{2}-\mathcal{A}_{1}\left(1+\mathscr{T} T^{*}\right)}{1+\mathcal{A}_{4} S_{0}}\right)\right] .
$$

We note that, aside from the denominator $1+\mathcal{A}_{4} S_{0}$, the components of the function $\Upsilon\left(S_{0}\right)$ are independent of $\hat{r}$. By identifying equation (41) as a Bernoulli-like ODE, we can use integrating factors and the matching conditions (33) to obtain the first-order non-linear autonomous differential equation for $S_{0}(\hat{r})$ :

$$
\frac{\partial S_{0}}{\partial \hat{r}}=-S_{0}\left(1-\sigma S_{0}\right) \sqrt{2 \int_{S_{0}}^{1} \frac{(1-\chi) \Upsilon(\chi)}{\chi(1-\sigma \chi)} d \chi} .
$$

It immediately follows that $S_{0}$ is monotone decreasing when $S_{0}$ lies between 0 and 1 . Hence, we conclude that $P$ and $T$ do not drastically change within the transition layer and the $O(\epsilon)$ perturbations $P_{1}$ and $T_{1}$ can be related to $S_{0}$, which is the solution of a first-order differential equation in $\hat{r}$.

2.3. Asymptotics of Region ii. While the leading-order dynamics of $S, T$, and $P$ have been determined in the transition layer, we still do not have an explicit form for $R(t)$ and $T^{*}(t)$. To find these, we now examine Region ii, where negligible water is present. From (1), we have that $S=0$ at $O\left(\epsilon^{-2}\right)$. However, this in turn causes a cascading effect in the asymptotic expansion of (1), and we conclude that $S=o\left(\epsilon^{n}\right)$ for all natural numbers $n$. Motivated by this fact, we anticipate that $S$ will be exponentially small in Region ii. Therefore, we can solve (1) explicitly, coupled with the condition that $S=1$ at $t=t^{*}$ :

$$
S=\left\{(1-\sigma) \exp \left[\frac{1}{\epsilon^{2}} \int_{t^{*}}^{t}\left(P_{S T}(T(r, s))-P(r, s)\right) \sqrt{\frac{1+\mathscr{T}}{1+\mathscr{T} T(r, s)}} d s\right]+\sigma\right\}^{-1} .
$$

If we then neglect exponentially small terms, (2) and (3) become

$$
\begin{aligned}
\frac{\partial}{\partial t}\left[\frac{P}{1+\mathscr{T} T}\right] & =\nabla \cdot\left[\frac{P \nabla P}{1+\mathscr{T} T}\right], \\
\frac{\partial T}{\partial t} & =\mathcal{A}_{3} \nabla^{2} T .
\end{aligned}
$$

For our boundary conditions in Region ii, we have the matching conditions (33)-(35), implying

$$
\begin{aligned}
\left.T\right|_{r \rightarrow R(t)} & \rightarrow T^{*}(t),\left.\quad P\right|_{r \rightarrow R(t)} \rightarrow P^{*}(t), \\
\left.\frac{\partial P}{\partial r}\right|_{r \rightarrow R(t)} & =\left.\frac{\partial P_{1}}{\partial \hat{r}}\right|_{\hat{r} \rightarrow+\infty} \rightarrow\left[\frac{1}{\delta}\left(\frac{1+\mathscr{T} T^{*}}{\left.(1+\mathscr{T}) P^{*}\right)}\right)-\sigma\right] R^{\prime}(t), \\
\left.\frac{\partial T}{\partial r}\right|_{r \rightarrow R(t)} & =\left.\frac{\partial T_{1}}{\partial \hat{r}}\right|_{\hat{r} \rightarrow+\infty} \rightarrow-\frac{1}{\mathcal{A}_{3}}\left[\mathcal{A}_{2}-\mathcal{A}_{1}\left(1+\mathscr{T} T^{*}\right)\right] R^{\prime}(t) .
\end{aligned}
$$

We must also give an initial condition for $R(t)$ and $T^{*}(t)$. As the drying front starts from the surface of the bean and the temperature is at the switching point of (6), the initial conditions are $R\left(t^{*}\right)=1$, $T^{*}\left(t^{*}\right)=T_{a}$. Finally, our solutions must also continue to agree with the external boundary conditions of the system, namely,

$$
\left.\frac{\partial T}{\partial r}\right|_{r=1}=\nu\left(\frac{1+\mathcal{A}_{4}}{1-\sigma}\right)\left[1-\left.T\right|_{r=1}\right] \quad \text { and }\left.\quad P\right|_{r=1}=P_{a}
$$

Therefore, our leading-order problem is a coupled system of two Stefan-like problems in a mass transfer setting, rather than the classical heat transfer setting [19]. As this coupled system of PDEs (45)-(50) is not explicitly solvable, we are motivated to use the large Stefan number approximation by considering the limiting case when $\delta \ll 1$. The large Stefan number approximation has been studied previously in the context of heat transfer on spheres (see e.g. [21]). For Region ii, the large Stefan limit $\delta \ll 1$ corresponds to the drying front moving very slowly relative to the speed of vapour diffusion. 
By rescaling time with $\tau=\delta\left(t-t^{*}\right)$, we can examine the asymptotic series

$$
T=T_{0}(r, \tau)+\delta T_{1}(r, \tau)+O\left(\delta^{2}\right), \quad P=P_{0}(r, \tau)+\delta P_{1}(r, \tau)+O\left(\delta^{2}\right)
$$

as $\delta \rightarrow 0^{+}$. In consequence, our leading-order Region ii problem (45)-(50) becomes

$$
\begin{gathered}
\nabla^{2} T_{0}=0,\left.\quad T_{0}\right|_{r \rightarrow R(\tau)} \rightarrow T^{*}(\tau),\left.\quad \frac{\partial T_{0}}{\partial r}\right|_{r \rightarrow R(\tau)} \rightarrow 0, \\
\nabla \cdot\left(\frac{P_{0} \nabla P_{0}}{1+\mathscr{T} T_{0}}\right)=0,\left.\quad P_{0}\right|_{r \rightarrow R(\tau)} \rightarrow P^{*}(\tau),\left.\quad \frac{\partial P_{0}}{\partial r}\right|_{r \rightarrow R(\tau)} \rightarrow\left(\frac{1+\mathscr{T} T^{*}}{(1+\mathscr{T}) P^{*}}\right) R^{\prime}(\tau), \\
\left.\frac{\partial T_{0}}{\partial r}\right|_{r=1}=\nu\left(\frac{1+\mathcal{A}_{4}}{1-\sigma}\right)\left[1-\left.T_{0}\right|_{r=1}\right],\left.\quad P_{0}\right|_{r=1}=P_{a}, \\
R(0)=1, \quad T^{*}(0)=T_{a} .
\end{gathered}
$$

Solving (52) implies that $T_{0} \equiv T^{*}(\tau)$ and applying (54) forces $T^{*}(\tau) \equiv 1$. In consequence, this reduces our coupled Stefan problem to a Stefan problem for pressure alone, i.e.

$$
\begin{aligned}
\nabla \cdot\left(P_{0} \nabla P_{0}\right)=0,\left.\quad P_{0}\right|_{r \rightarrow R(\tau)} & \rightarrow 1,\left.\quad P_{0}\right|_{r=1}=P_{a}, \\
\left.\frac{\partial P_{0}}{\partial r}\right|_{r \rightarrow R(\tau)} & \rightarrow R^{\prime}(\tau), \\
R(0) & =1 .
\end{aligned}
$$

We note that this solution cannot satisfy the initial condition (55) for $T^{*}(t)$. For this to be resolved, we would have to consider the full problem in $t,(45)-(50)$, which is not readily solvable.

2.3.1. Determining $R(t)$ in Cartesian Co-ordinates with $T^{*} \equiv 1$. In the limiting case where $\delta \ll 1$, i.e. $T^{*} \equiv 1$, we can solve (56), provided that we neglect any short-time discrepancies between the initial condition $T^{*}(0)=T_{a}$ and $T^{*} \equiv 1$. Solving this PDE system (56) gives us

$$
P_{0}(r, \tau)=\sqrt{1-\left(1-P_{a}^{2}\right)\left(\frac{r-R(\tau)}{1-R(\tau)}\right)}
$$

and our Stefan condition (57) gives us the ODE

$$
\frac{d R}{d \tau}=-\frac{1-P_{a}^{2}}{2(1-R)} .
$$

Based on the initial condition from (58), our drying front in Cartesian co-ordinates based on leading-order asymptotics, $R_{\text {Cart }}(\tau)$, is

$$
R_{\text {Cart }}(\tau)=1-\sqrt{\left(1-P_{a}^{2}\right) \tau} .
$$

By returning to the original timescale of Region ii, we determine that $P_{0}$ can be fully expressed in Cartesian co-ordinates as

$$
P_{0}^{\text {Cart }}(r, t)=\sqrt{P_{a}^{2}+(1-r) \sqrt{\frac{1-P_{a}^{2}}{\delta\left(t-t_{\text {Cart }}^{*}\right)}}} .
$$

Finally, we determine from (61) that the time to completely dry a bean based on leading-order asymptotics is

$$
t_{\text {Cart }}^{\mathrm{dry}}=t_{\text {Cart }}^{*}+\frac{1}{\delta\left(1-P_{a}^{2}\right)} .
$$

Using parameter values shown in [6], as well as typical values $P_{a}=0.0879, \delta=0.1011, \sigma=0.0842$, and $t_{\text {Cart }}^{*} \approx 0.494$, we compute that $t_{\text {Cart }}^{\text {dry }} \approx 10.46$, or about 2768 seconds in dimensional units. 
2.3.2. Determining $R(t)$ in Spherical Co-ordinates with $T^{*} \equiv 1$. In the limiting case where $\delta \ll 1$, i.e. $T^{*} \equiv 1$, we have that in spherical co-ordinates, by solving (56),

$$
P_{0}(r, \tau)=\sqrt{1-\left(\frac{1-P_{a}^{2}}{r}\right)\left(\frac{r-R(\tau)}{1-R(\tau)}\right)},
$$

and our Stefan condition (57) gives us the ODE

$$
\frac{d R}{d \tau}=-\frac{1-P_{a}^{2}}{2 R(1-R)} .
$$

We use our initial condition (58) to give us, in implicit form, that the inverse function of the drying front in spherical co-ordinates, $\tau_{\mathrm{Sph}}(R)$, satisfies the equation

$$
\tau_{\mathrm{Sph}}(R)=\frac{1-R^{2}(3-2 R)}{3\left(1-P_{a}^{2}\right)} .
$$

We can invert (66) and solve $R_{\mathrm{Sph}}(\tau)$ in the correct domain and range:

$$
R_{\mathrm{Sph}}(\tau)=\frac{1}{2}\left(1-\frac{\exp \left(\frac{2 \pi i}{3}\right)}{\Xi\left(3\left(1-P_{a}^{2}\right) \tau\right)}-\exp \left(\frac{-2 \pi i}{3}\right) \Xi\left(3\left(1-P_{a}^{2}\right) \tau\right)\right),
$$

where

$$
\Xi(\chi)=\sqrt[3]{2 \sqrt{\chi(\chi-1)}-2 \chi+1}
$$

and $\Xi(\chi)$ uses the principal branch of the cube root. Now that we have determined $R(\tau)$ in spherical coordinates, we can return to our original timescale of the problem and obtain that our leading-order asymptotic approximation for $P$ is

$$
\tilde{P}_{0}^{\mathrm{Sph}}(r, t)=\sqrt{1-\left(\frac{1-P_{a}^{2}}{r}\right)\left(1-\frac{2(1-r)}{1+\frac{\exp \left(\frac{2 \pi i}{3}\right)}{\Xi\left(3 \delta\left(1-P_{a}^{2}\right)\left(t-t_{\mathrm{Sph}}^{*}\right)\right.}+\exp \left(\frac{-2 \pi i}{3}\right) \Xi\left(3 \delta\left(1-P_{a}^{2}\right)\left(t-t_{\mathrm{Sph}}^{*}\right)\right)}\right)}
$$

To determine the time where the bean becomes fully dry, we substitute $R=0$ into (66) to obtain, in our original timescale, that

$$
t_{\mathrm{Sph}}^{\mathrm{dry}}=t_{\mathrm{Sph}}^{*}+\frac{1}{3 \delta\left(1-P_{a}^{2}\right)} .
$$

Therefore, to leading order, the time for a spherical coffee bean to dry out completely is $t_{\mathrm{Sph}}^{\mathrm{dry}} \approx 3.495$, or about 925 seconds in dimensional units. Figure 2(a) shows a comparison between the Cartesian and spherical asymptotic approximations of $R(t)$.

2.4. Comparison of Asymptotic Approximations with Numerical Results. We now compare these asymptotic approximations with the numerical solution of the PDE system (1)-(3), considering the drying front $R(t)$ in particular. We solve the the PDE system (1)-(3) in MATLAB using the method of lines and a second-order central finite difference scheme in space. We use a stiff adaptive ODE solver in time, namely the MATLAB function ode15s, to achieve convergence. As we can see in Figure 2(b), the general shape of the dimensional drying front $R(t)$ agrees reasonably well with the dimensional drying front seen in the numerical solution, especially as $R(t) \rightarrow 0$. However, we also see that the drying time in the numerical solution is larger than the predicted $t_{\mathrm{Sph}}^{\mathrm{dry}}$ from asymptotic results. This is to be expected, as the asymptotic results used were for when the Stefan number $\frac{1}{\delta} \rightarrow+\infty$. Therefore, for a smaller (but still large) Stefan number, we expect the drying time to be longer. Additionally, these approximations for the drying front $R(t)$ assume $T^{*} \equiv 1$. Because $T^{*}(t)$ is less than unity in the numerical simulations, this will cause the drying front to be slower than the asymptotic approximation, which can explain why the numerical solution takes longer to dry out the entire bean. 


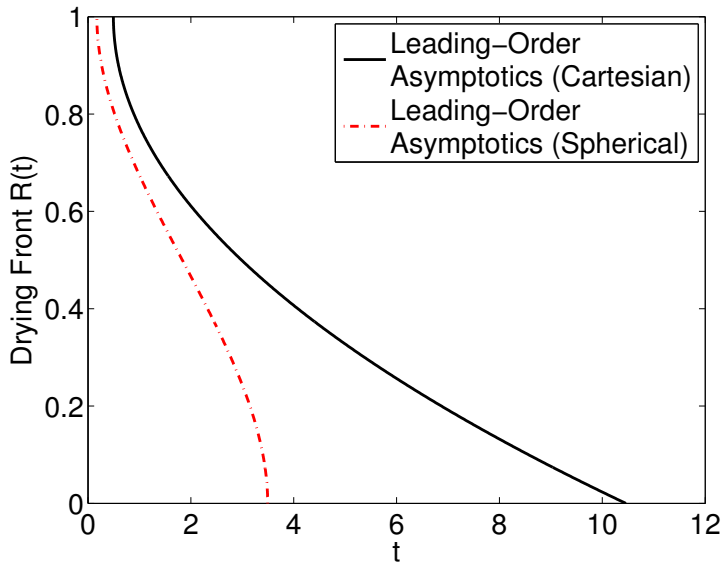

(a)

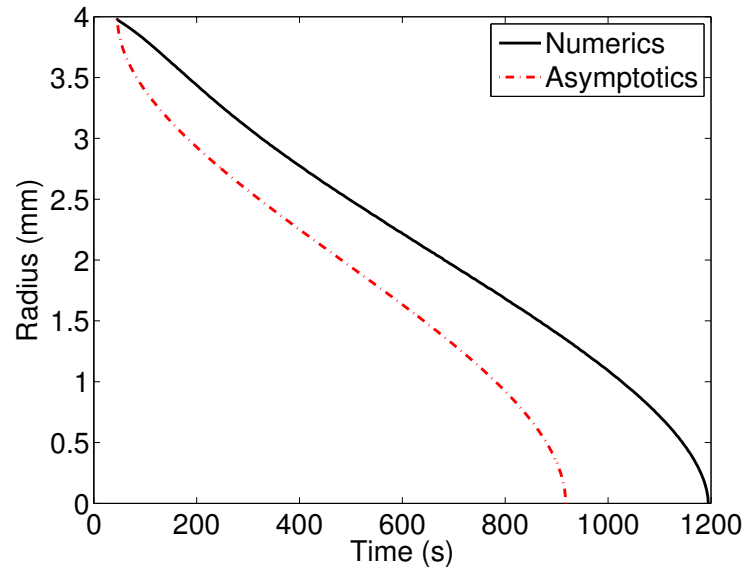

(b)

FIG. 2. Comparison of predictions of the drying front position $R(t)$ for the variable temperature regime. (a) Predictions $R_{\text {Cart }}(t)$ from (61) and $R_{S p h}(t)$ from (67). (b) Spherical predictions $R_{S p h}(t)$ from (67), shown in dash-dot red, and numerical solutions of (1)-(8), shown in black. All predictions in (b) are shown in dimensional units.

3. Asymptotics of the Multiphase Model with Constant Temperature. In Section 2, we have given an analysis of the leading-order equations governing Region i, Region ii, and the transition layer. However, the numerical solutions indicate the thermal timescale of the multiphase model is much smaller than the vapour diffusive timescale. In consequence, it seems reasonable to examine a reduced model where the coffee bean is at the externally imposed roasting temperature throughout. Additionally, many of the leading-order equations can be solved explicitly if the temperature is spatially uniform and this helps in interpreting the behaviour of the moisture content and vapour pressure. Therefore, we are motivated from a physical and an asymptotics point of view to consider a reduced model with $T \equiv 1$.

In this section, we assume that $T \equiv 1$ throughout the bean, which this means that the transition region is created immediately (i.e. $\left.t^{*}=0\right)$ and the system of PDEs (1)-(2) become

$$
\begin{gathered}
\frac{\partial S}{\partial t}=-\frac{1}{\epsilon^{2}}(1-P) S(1-\sigma S), \\
\frac{\partial}{\partial t}[(1-\sigma S) P]=\frac{1}{\delta \epsilon^{2}}(1-P) S(1-\sigma S)+\nabla \cdot(P \nabla P),
\end{gathered}
$$

with boundary conditions

$$
\left.P\right|_{r=1}=P_{a},\left.\quad \frac{\partial P}{\partial r}\right|_{r=0}=0,
$$

and initial conditions

$$
S(r, 0)=1, \quad P(r, 0)=1 .
$$

Formally, we will consider the asymptotics of this system in the limit as $\epsilon \rightarrow 0^{+}$. We will take $\delta=O(1)$ except where significant simplification is found by taking $\delta \ll 1$, and all other parameters are assumed to be $O(1)$.

3.1. Asymptotics in Region i. In Region $\mathrm{i}$, we have that $P=1$ to leading order. In fact, we note that $P, S \equiv 1$ is the exact solution in Region i, as these constant solutions satisfy both PDEs, the initial conditions, and the symmetry condition in $P$ at $r=0$. It is important to note that, since we assume that Region $\mathrm{i}$ is never in contact with the surface of the bean, the boundary condition at $r=1$ does not apply.

3.2. Asymptotics of the Transition Layer. As in Section 2, we introduce the scaling $r=R(t)+\epsilon \hat{r}$ to examine the behaviour as $S$ transitions from 1 to 0 . Again, we can expand $P$ and $S$ as asymptotic series as $\epsilon \rightarrow 0$ :

$$
S=S_{0}(\hat{r}, t)+\epsilon S_{1}(\hat{r}, t)+O\left(\epsilon^{2}\right), \quad P=1+\epsilon P_{1}(\hat{r}, t)+O\left(\epsilon^{2}\right) .
$$



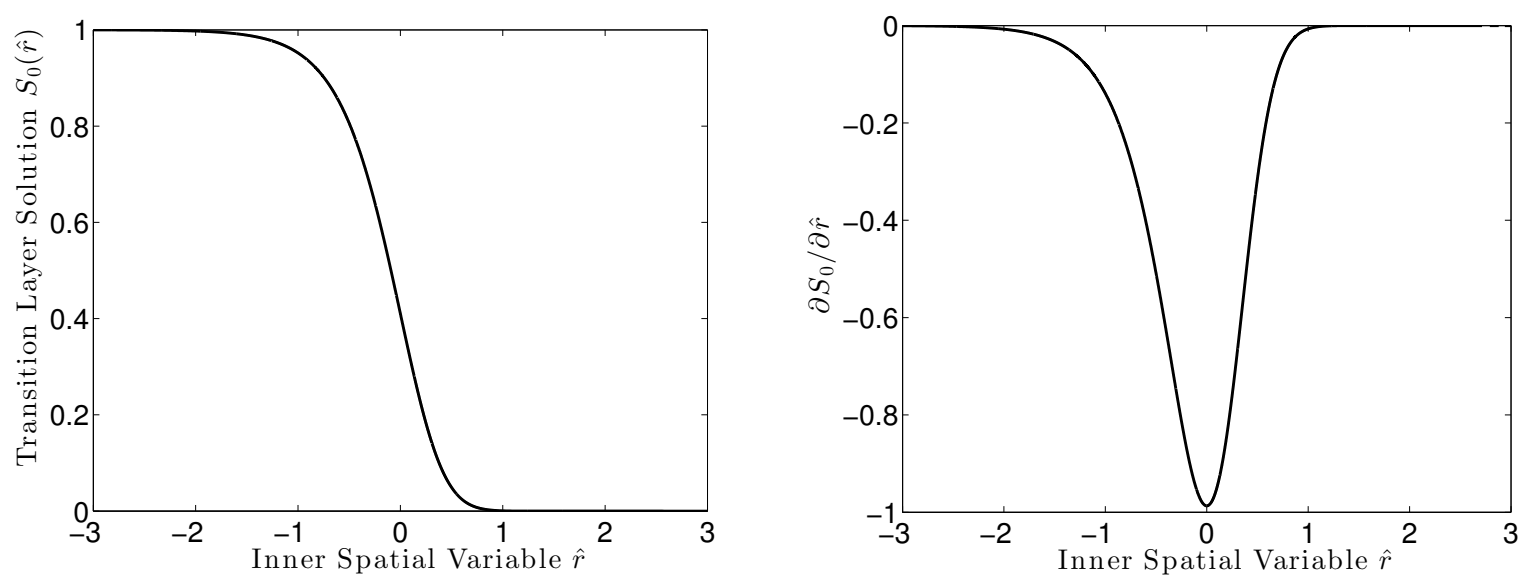

FIG. 3. Numerical solution of the ODE (77). The left panel shows the solution $S_{0}(\hat{r})$ and the right panel shows its spatial derivative $\frac{\partial S_{0}}{\partial \hat{r}}$. For uniqueness, we pick a constant of integration so that $S_{0}(\hat{r})$ has an inflection point at $r=0$.

Noting that temperature is constant (implying that $T_{1} \equiv 0$ and $T^{*} \equiv 1$ ), the equation (42) for $\Upsilon\left(S_{0}\right)$ reduces to $\Upsilon\left(S_{0}\right) \equiv \frac{1}{\delta}-\sigma$. From (37), this gives us

$$
\frac{\partial P_{1}}{\partial \hat{r}}=\left(\frac{1}{\delta}-\sigma\right) R^{\prime}(t)\left(1-S_{0}\right)
$$

and from (43), gives us the first-order non-linear autonomous ODE:

$$
\frac{\partial S_{0}}{\partial \hat{r}}=-S_{0}\left(1-\sigma S_{0}\right) \sqrt{2\left(\frac{1}{\delta}-\sigma\right)\left[\frac{1-\sigma}{\sigma} \log \left(\frac{1-\sigma}{1-\sigma S_{0}}\right)+\log \left(\frac{1}{S_{0}}\right)\right]} .
$$

with matching conditions $S_{0} \rightarrow 0$ as $\hat{r} \rightarrow+\infty$ and $S_{0} \rightarrow 1$ as $\hat{r} \rightarrow-\infty$. It is important to note a few key points about equation (77). Firstly, it is not explicitly solvable. Secondly, due to translational invariance, we require an additional constraint for uniqueness. This can be achieved, for example, by assuming the unique inflection point of $S_{0}$ occurs at $r=0$. With this additional constraint, we numerically solve (77) and plot the results in Figure 3.

3.3. Asymptotics in Region ii. In Region ii, we have, from (71), that $S=0$ at $O\left(\epsilon^{-2}\right)$, which again causes a cascading effect in the asymptotic expansion of (71). Similar to what was done in Section 2, we find that $S$ is exponentially small in Region ii and is given by

$$
S=\left\{(1-\sigma) \exp \left[\frac{1}{\epsilon^{2}}\left(t-\int_{0}^{t} P(r, s) d s\right)\right]+\sigma\right\}^{-1} .
$$

Additionally, by neglecting exponentially small $S$, (72) becomes

$$
\frac{\partial P}{\partial t}=\nabla \cdot(P \nabla P)
$$

From (76), our boundary and initial conditions become

$$
\left.P\right|_{r=1}=P_{a},\left.\quad P\right|_{r \rightarrow R(t)} \rightarrow 1,\left.\quad \frac{\partial P}{\partial r}\right|_{r \rightarrow R(t)} \rightarrow\left(\frac{1}{\delta}-\sigma\right) R^{\prime}(t), \quad R(0)=1 .
$$

This problem has a similarity solution in Cartesian co-ordinates, as will be shown in Section 3.3.1, although it cannot be explicitly solved. However, we can also examine the physically relevant large Stefan-number limit by letting $\delta \rightarrow 0^{+}$, as was done in Section 2.3. By rescaling time with $\tau=\delta t$ and considering the asymptotic series $P \sim P_{0}(r, \tau)+\delta P_{1}(r, \tau)+O\left(\delta^{2}\right)$, (79)-(80) become

$$
\nabla \cdot\left(P_{0} \nabla P_{0}\right)=0,\left.\quad P_{0}\right|_{r=1}=P_{a},\left.\quad P_{0}\right|_{r \rightarrow R(\tau)} \rightarrow 1,\left.\quad \frac{\partial P_{0}}{\partial r}\right|_{r \rightarrow R(\tau)} \rightarrow R^{\prime}(t), \quad R(0)=1 .
$$


Solving (81) like in Section 2, we determine that in Cartesian co-ordinates,

$$
P_{0}(r, t)=\sqrt{P_{a}^{2}+(1-r) \sqrt{\frac{1-P_{a}^{2}}{\delta t}}}, \quad R(t)=1-\sqrt{\left(1-P_{a}^{2}\right) \delta t},
$$

and in spherical co-ordinates,

$$
\begin{aligned}
\tilde{P}_{0}(r, t) & =\sqrt{1-\left(\frac{1-P_{a}^{2}}{r}\right)\left[1-\frac{2(1-r)}{\left.1+\frac{\exp \left(\frac{2 \pi i}{3}\right)}{\Xi\left(3\left(1-P_{a}^{2}\right) \delta t\right)}+\frac{\Xi\left(3\left(1-P_{a}^{2}\right) \delta t\right)}{\exp \left(\frac{2 \pi i}{3}\right)}\right]},\right.} \\
R(t) & =\frac{1}{2}\left(1-\frac{\exp \left(\frac{2 \pi i}{3}\right)}{\Xi\left(3\left(1-P_{a}^{2}\right) \delta t\right)}-\frac{\Xi\left(3\left(1-P_{a}^{2}\right) \delta t\right)}{\exp \left(\frac{2 \pi i}{3}\right)}\right),
\end{aligned}
$$

where $\Xi(\chi)=\sqrt[3]{2 \sqrt{\chi(\chi-1)}-2 \chi+1}$.

3.3.1. Determining $R(t)$ in Cartesian Co-ordinates using Similarity Solutions. One might consider using a similarity solution to solve the system (79)-(80) in Cartesian coordinates without the assumption that $\delta \ll 1$. To do this, we let $P=h(\eta)$, where $\eta=\frac{1-r}{\sqrt{t}}$. Substituting this transformation into (79) gives

$$
\left(h(\eta) h^{\prime}(\eta)\right)^{\prime}+\frac{\eta}{2} h^{\prime}(\eta)=0,
$$

and $(80)$ becomes

$$
h(0)=P_{a}, \quad h(\lambda)=1, \quad h^{\prime}(\lambda)=\frac{\lambda(1-\delta \sigma)}{2 \delta} .
$$

Here, $\eta=\lambda$ corresponds to the moving boundary $R(t)$. Thus, our drying front based on the Cartesian similarity solution, is given by

$$
R_{\mathrm{SS}}(t)=1-\lambda \sqrt{t} .
$$

We note that our choice of $\eta$ allows us to automatically satisfy the initial condition $R(0)=1$ and we can determine from this equation when the bean will be completely dry, i.e. when $R_{\mathrm{SS}}(t)=0$. This gives us $t_{S S}^{\mathrm{dry}}=\frac{1}{\lambda^{2}}$. As (84) is not explicitly solvable, it is necessary to numerically solve this boundary value problem in order to determine $\lambda$. Using the shooting method, with the typical values $P_{a}=0.0879, \delta=0.1011$, and $\sigma=0.0842$, we find that $\lambda \approx 0.3152$, implying that $t_{S S}^{\mathrm{dry}} \approx 10.06$, or about 2664 seconds in dimensional units. With less than a $1 \%$ relative error to $t_{S S}^{\text {dry }}$, we conclude that $t_{\text {Cart }}^{\text {dry }} \approx 9.964$, as described in (63), is a very good approximation to the drying time computed from the similarity solution. Figure 4(a) shows a comparison of the drying front $R_{\mathrm{SS}}(t)$ with the Cartesian drying front determined previously via asymptotic methods, namely, $R_{\text {Cart }}(t)$ given in (61).

3.4. Comparison of Asymptotic Approximations with Numerical Results. Comparing the various asymptotic approximations with the numerical solutions of (71)-(74), we can see in Figure 4(b) that the general shape of the dimensional drying front $R(t)$ agrees well with the dimensional drying front seen in the numerical solution. Because we have assumed that $T^{*} \equiv 1$, we no longer have differences induced by varying the temperature that were seen Section 2. Therefore, it is expected that the drying front $R(t)$ determined via asymptotics fits closer to the numerics. We see, like in Section 2, that the drying time predicted by the numerical solution is larger than $t_{\mathrm{Sph}}^{\mathrm{dry}}$ determined from asymptotic results in (70). However, this is to be expected; a large (but finite) Stefan number would cause the drying time to be longer than the time produced by the limit $\delta \rightarrow 0^{+}$. This is confirmed by comparing the drying front in the full numerical solution with the drying front obtained by solving (79)-(80) numerically. Indeed, by allowing $\epsilon \rightarrow 0^{+}$, we only observe significant discrepancies between these two drying fronts near the surface and the centre of the bean. This is to be expected, as the transition layer will be on a semi-infinite region in both of these cases and will have different behaviour due to the boundary conditions. 


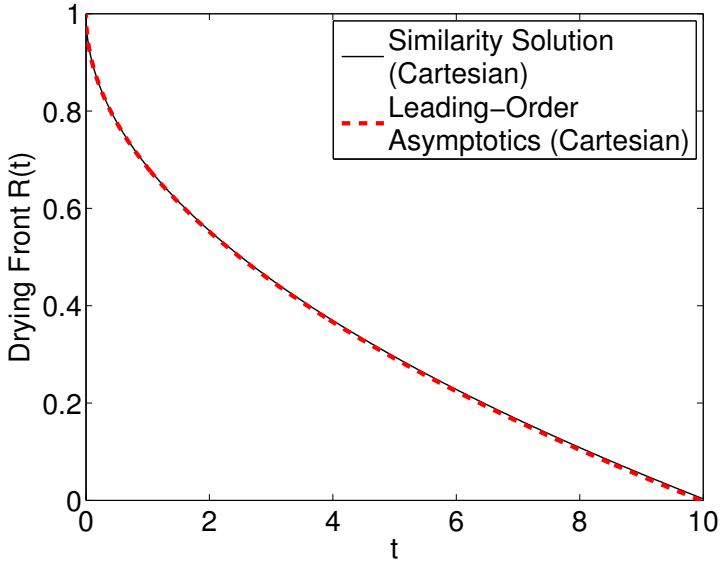

(a)

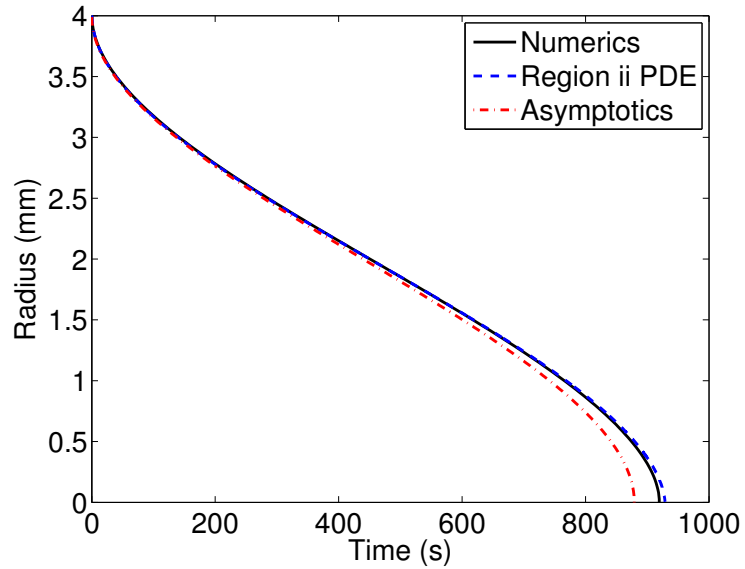

(b)

FIG. 4. Comparison of predictions of the drying front position $R(t)$ for the constant temperature regime. (a) Cartesian predictions $R_{S S}(t)$ from (86) and $R_{\text {Cart }}(t)$ from (82). (b) Spherical predictions $R_{S p h}(t)$ from (83), shown in dash-dot red, and numerical solutions of (71)-(74), shown in black, and of (79)-(80), shown in dashed blue. All predictions in (b) are shown in dimensional units.

4. Discussion. In this paper, we have extended results of the "simplified" form of the multiphase model presented in [6] via asymptotic methods, in order to better understand the qualitative features of the coffee bean roasting process. Motivated by previous numerical results, we considered the limit $\epsilon \rightarrow 0^{+}$, representing the situation where the rate of vapour transport by Darcy flow is much smaller than the evaporation rate. The asymptotic analysis showed that the solution could be divided into two main regions and a transition layer. The entire bean was in the first region until a time $t^{*}$, when a thin transition layer appears at the surface of the bean. This transition layer then propagated into the bean creating a second main region between it and the surface of the bean. This asymptotic limit is different from what has been studied previous in drying models, since the rigid cellulose structure of the solid coffee bean creates a large build-up of vapour pressure that drives the vapour to the external environment. The analysis shows that a narrow drying front, represented by the transition layer, is crucial to the drying process in this limit.

In the first region, the vapour pressure is in equilibrium with the steam table pressure and the moisture content of the bean remains at its initial value, with heat flow governed by the heat equation. In the thin transition region, the moisture content changes rapidly from its initial value to a small value. Here, evaporation dominates and the temperature and vapour pressure remain spatially uniform. Finally, in the second main region, there is almost no water and therefore no evaporation. The problem in this second region consists of diffusion equations for the heat and vapour flow with coupling through the matching conditions, similar to a Stefan problem, at the transition layer.

Numerical simulations suggest that the externally applied roasting temperature is attained globally fairly quickly; hence, the case where temperature is fixed at the roasting temperature was considered. This also allowed the coupled Stefan problem to be reduced to a single Stefan problem, which could then be solved via similarity solutions or large Stefan number asymptotics. The leading order expressions are shown to agree well with the dynamics of the drying front found from numerical simulations, under both spherical and planar geometries.

Despite several simplifications made in obtaining asymptotic solutions in each of the regions of the coffee bean, a reasonable agreement between the asymptotic approximations and the numerical solution of the multiphase model as described in [6] has been obtained. This suggests that the asymptotics found here accurately capture the qualitative behaviour of the coffee bean roasting process, and provide an acceptable compromise between a simpler heat transfer model (such as those presented in [5]) and more complicated multiphase models. The asymptotic results presented in this paper can be extended in order to determine the asymptotic dynamics of related heat and mass transfer models. The complete multiphase model described in [6] incorporates variable porosity, and by using similar methods to those shown here, one might determine the leading-order behaviour of the multiphase model with variable porosity. Additionally, the analysis on more complicated geometries, such as non-radially symmetric perturbations of a slab or sphere, may lead to 
destabilising the "drying front" observed in simpler geometries. Similarly, one might use the general asymptotic results for the multiphase model discussed here to guide the development of relevant solid mechanics models, which take into account the structural properties of the coffee bean and allow for variations in coffee quality due to structural deformations which may occur during heating and roasting. Asymptotic results may also guide in the development of more complicated models involving many more chemical reactions, as well as in understanding taste and aromatic properties of the final product.

Appendix A. Derivation of the Multiphase Model. Here, we will derive the multiphase model (71)-(8) discussed in this paper, starting with equations (42)-(49) in [6]. Using the rescalings

$$
S=\sigma \hat{S}, \quad T=\hat{T}, \quad p_{v}=p_{S T}(1) \hat{P}, \quad t=\frac{\phi}{\mathscr{D}_{3} p_{S T}(1)} \hat{t},
$$

and defining the parameters

$$
\begin{aligned}
\epsilon & =\frac{\sqrt{\mathscr{D}_{3} \sqrt{1+\mathscr{T}}}}{\phi}, \quad \delta=\frac{p_{S T}(1) \alpha_{2}}{\sigma(1+\mathscr{T})}, \quad \beta=\frac{B_{2} \mathscr{T}}{1+\mathscr{T}}, \\
\mathcal{A}_{1} & =\frac{\sigma \phi}{\alpha_{1} \mathscr{C}_{1}(1-\phi) \mathscr{T}}, \quad \mathcal{A}_{2}=\gamma \mathcal{A}_{1}, \quad \mathcal{A}_{3}=\frac{\phi\left(\zeta_{1}(1-\phi)+\zeta_{3} \phi\right)}{\mathscr{D}_{3} p_{S T}(1) \alpha_{1} \mathscr{C}_{1}(1-\phi)}, \quad \mathcal{A}_{4}=\frac{\phi \sigma\left(\zeta_{2}-\zeta_{3}\right)}{\zeta_{1}(1-\phi)+\zeta_{3} \phi},
\end{aligned}
$$

the model of [6] becomes (dropping hats)

$$
\begin{aligned}
\frac{\partial S}{\partial t} & =-\frac{1}{\epsilon^{2}} I_{v} \\
\frac{\partial}{\partial t}\left[\frac{(1+\mathscr{T}) P(1-\sigma S)}{1+\mathscr{T} T}\right] & =-\frac{1}{\delta} \frac{\partial S}{\partial t}+\nabla \cdot\left[\frac{(1+\mathscr{T}) P \nabla P}{1+\mathscr{T} T}\right], \\
\frac{\partial T}{\partial t}+\mathcal{A}_{1} \frac{\partial}{\partial t}[S(1+\mathscr{T} T)] & =\mathcal{A}_{2} \frac{\partial S}{\partial t}+\mathcal{A}_{3} \nabla \cdot\left[\left(1+\mathcal{A}_{4} S\right) \nabla T\right] .
\end{aligned}
$$

Here, the rescaled evaporation rate $I_{v}$ and the rescaled steam table pressure $P_{S T}(T)$ are given by

$$
I_{v}=S(1-\sigma S)\left(P_{S T}-P\right) \sqrt{\frac{1+\mathscr{T}}{1+\mathscr{T} T}} \quad \text { and } \quad P_{S T}(T)=\exp \left(\frac{\beta(T-1)}{1+\mathscr{T} T}\right)
$$

We note that in [6], the initial saturation value was defined as $S_{0}$. This notation means something different in the analysis of this paper, so we have therefore changed the notation of the initial saturation to be denoted as $\sigma$. The boundary conditions we impose on the PDE system (1)-(3) are the symmetry conditions at the centre of the bean

$$
\nabla T \cdot \mathbf{n}=0, \quad \nabla P \cdot \mathbf{n}=0 \text { at } r=0,
$$

as well as the heat transfer condition

$$
\nabla T \cdot \mathbf{n}=\nu\left(\frac{1-\sigma S}{1-\sigma}\right)\left(\frac{1+\mathcal{A}_{4}}{1+\mathcal{A}_{4} S}\right)(1-T) \text { at } r=1,
$$

where

$$
\nu=\frac{\mathrm{Nu}_{v} \zeta_{3} \phi(1-\sigma)}{\left(\zeta_{1}(1-\phi)+\zeta_{3} \phi\right)\left(1+\mathcal{A}_{4}\right)} .
$$

Previously, the model introduced in [6] imposes a Dirichlet condition in $P$ at the surface of the bean. We will instead impose a different boundary condition for $P$ in order to prevent condensation from occurring at the surface of the bean. This can be achieved by imposing that $P$ equals the steam table pressure for temperatures below the evaporating temperature, i.e.

$$
\left.P\right|_{r=1}= \begin{cases}P_{S T}(T), & T<T_{a} \\ P_{a}, & T \geq T_{a}\end{cases}
$$


Here, $P_{a}:=\frac{1+\mathscr{T}}{p_{S T}(1)}$ and $T_{a}:=P_{S T}^{-1}\left(P_{a}\right)$. We will also make the assumption that the switching between the boundary condition for $P$ only occurs at one critical time, namely, $t^{*}$. We define $t^{*}$ as the time when the surface temperature equals the evaporation temperature $T_{a}$, i.e. as the solution to the equation $T\left(1, t^{*}\right)=T_{a}$. Finally, we impose the initial conditions corresponding to uniform initial moisture content, room temperature, and equilibrium steam table pressure, i.e.

$$
S(r, 0)=1, \quad T(r, 0)=0, \quad P(r, 0)=P_{S T}(0) .
$$

\section{REFERENCES}

[1] J.M. Talbot, Grounds for agreement: The political economy of the coffee commodity chain. Rowman and Littlefield, Oxford, UK (2004).

[2] J. Baggenstoss, Coffee roasting and quenching technology - Formation and stability of aroma compounds. PhD thesis, Swiss Federal Institute of Technology (2008).

[3] S. Schenker, Investigations on the hot air roasting of coffee, PhD thesis, Swiss Federal Institute of Technology, Zurich (2000).

[4] X. Wang and L.T. Lim, A kinetics and modeling study of coffee roasting under isothermal conditions, Food and Bioprocess Technology, 7 (2014), pp. 621-632.

[5] A. Fabbri, C. Cevoli, L. Alessandrini, and S. Romani, Numerical modeling of heat and mass transfer during coffee roasting process, Journal of Food Engineering, 105 (2011), pp. 264-269.

[6] N.T. Fadai, J. Melrose, C.P. Please, A. Schulman, R.A. Van Gorder, A Heat and Mass Transfer Study of Coffee Bean Roasting, International Journal of Heat and Mass Transfer 104 (2017), 787-799.

[7] K.M. Moroney, W.T. Lee, S.B.G. O'Brien, F. Suijver, J. Marra, Modelling of coffee extraction during brewing using multiscale methods: an experimentally validated model, Chemical Engineering Science, 137 (2015), pp. 216-234.

[8] W.N. Hernandez-Diaz, I.I. Ruiz-Lopez, M.A. Salgado-Cervantes, G.C. Rodriguez-Jimenez, and M.A. Garcia-Alvarado, Modeling heat and mass transfer during drying of green coffee beans using prolate spheroidal geometry, Journal of Food Engineering, 86 (2008), pp. 1-9.

[9] A. Halder, A. Dhall, and A.K. Datta, Modeling transport in porous media with phase change: applications to food processing, Journal of Heat Transfer, 133 (2011), 031010.

[10] H. Ni and A.K. Datta, Heat and moisture transfer in baking of potato slabs, Drying Technology 17 (1999), pp. $2069-2092$.

[11] A. Dhall, A. Halder, and A.K. Datta, Multiphase and multicomponent transport with phase change during meat cooking, Journal of Food Engineering, 113 (2012), pp. 299-309.

[12] Y. Llave, K. Takemori, M. Fukuoka, T. Takemori, H. Tomita, and N. Sakai, Mathematical modeling of shrinkage deformation in eggplant undergoing simultaneous heat and mass transfer during convection-oven roasting, Journal of Food Engineering, 178 (2016), pp. 124-136.

[13] H. Huang, P. Lin, and W. Zhou, Moisture transport and diffusive instability during bread baking, SIAM Journal on Applied Mathematics, 68 (2007), pp. 222-238.

[14] J Chen, K Pitchai, S Birla, M Negahban, D Jones, J Subbiah, Heat and Mass Transport during Microwave Heating of Mashed Potato in Domestic Oven-Model Development, Validation, and Sensitivity Analysis, Journal of Food Science 79, pp. E1991-E2004.

[15] J. Zhang and A.K. Datta, Mathematical modeling of bread baking process, Journal of Food Engineering, 75 (2006), pp. 78-89.

[16] M. A. Stanish, G. S. Schajer, and F. Kayihan, A Mathematical Model of Drying for Hygroscopic Porous Media, AIChE Journal, 32 (1986), pp. 1301-1311.

[17] S. K. Truscott and I. W. Turner, A heterogeneous three-dimensional computational model for wood drying, Applied Mathematical Modelling, 29 (2005), pp. 381-410.

[18] P. Chen and D. C. T. Pei, A Mathematical Model of Drying Processes, International Journal of Heat and Mass Transfer, 32 (1989), pp. 297-310.

[19] A. M. Meirmanov, The Stefan Problem, De Gruyter Expositions in Mathematics, Berlin: De Gruyter (1992).

[20] I. Langmuir, The Vapor Pressure of Metallic Tungsten, Physical Review, 2 (1913), pp. 329-342.

[21] S. W. McCue, B. Wu, J. M. Hill, Classical two-phase Stefan problem for spheres, Proceedings of the Royal Society A 464 (2008), pp. 2055-2076. 